\title{
Endovascular treatment of irregular and complicated intracranial aneurysms with coils using double microcatheter technique
}

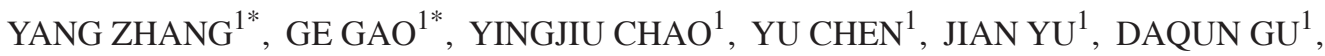 \\ JIANJUN WEI ${ }^{1}, \mathrm{CHAOSHI} \mathrm{NIU}^{1-3}$ and XIANMING FU ${ }^{1,2}$ \\ ${ }^{1}$ Department of Neurosurgery, Anhui Provincial Hospital; ${ }^{2}$ Anhui Provincial Stereotactic Neurosurgical Institute; \\ ${ }^{3}$ Anhui Province Key Laboratory of Brain Function and Brain Disease, Hefei, Anhui 230001, P.R. China
}

Received May 19, 2016; Accepted October 3, 2016

DOI: $10.3892 /$ etm.2016.3960

\begin{abstract}
Embolisation of irregular and complicated aneurysms is a great challenge for neuroradiologists. To overcome possible complications of endovascular treatment of these type of aneurysms, methods such as intracranial stents, balloon remodelling, and the double microcatheter technique have been developed. The aim of the study was to report our preliminary experience with endovascular treatment of irregular and complicated intracranial aneurysm with coils using double microcatheter technique, and evaluation of its feasibility and clinical advantages. In this retrospective study, 37 cases diagnosed with irregular and complicated intracranial aneurysms and treated using double microcatheter method from July, 2013 to May, 2015, were followed up for six months after discharge. All the aneurysms were successfully embolized using the double microcatheter technique. Immediate post-embolization angiography showed no residual contrast filling in 35 cases, and residual filling in 2 cases. At discharge, according to the modified Rankin Scale score, all the patients recovered without any complications or death. A follow-up of 24 cases with digital subtraction angiography for 6 months revealed no recanalization. In conclusion, the double microcatheter technique for irregular intracranial aneurysms is effective and simple with few complications.
\end{abstract}

\section{Introduction}

Although complicated intracranial aneurysms have not been defined thus far, they generally refer to giant, wide-necked, tiny, irregular-shaped, dissecting, fusiform and false aneurysms (1). Irregular aneurysm is a complicated aneurysm that

Correspondence to: Dr Yang Zhang, Department of Neurosurgery, Anhui Provincial Hospital, 17 Lujiang Road, Hefei, Anhui 230001, P.R. China

E-mail: be2733039liang@163.com

*Contributed equally

Key words: intracranial aneurysm, double microcatheter technique, endovascular treatment is refractory to treatment with interventional embolization (2). Balloon remodeling or stenting does not adequately embolize aneurysms.

Nonetheless, the Department of Neurosurgery at Anhui Provincial Hospital treated 37 cases of complicated intracranial aneurysms with coils using double microcatheter technique between July 2013 and May 2015 with satisfactory results. Thus, this method is effective as it poses few complications.

\section{Patients and methods}

General data. Between July 2013 and May 2015, based on a definitive diagnosis using digital subtraction angiography (DSA), the Department of Neurosurgery at Anhui Provincial Hospital performed interventional therapy with coils using the double microcatheter technique on 37 cases of irregular intracranial aneurysms, in 13 males and 24 females, aged 42-78 years (average, 57.2 years). Three of these cases were unruptured and the remaining 34 were ruptured. At admission, there were 8 cases of Hunt-Hess grade I, 19 grade II, 9 grade III, and 1 grade IV. The aneurysms were distributed as follows: 9 cases of anterior communicating artery aneurysms, 13 posterior communicating artery aneurysm, 3 carotid-ophthalmic aneurysms, 2 anterior choroidal artery segmental aneurysms, 3 aneurysms at the origin of PICA, 5 middle cerebral artery bifurcation aneurysms, and 2 basilar artery apex aneurysms.

Treatment protocol. The patient was placed in supine position, under general anesthesia, followed by catheterization of the right femoral artery using $6 \mathrm{~F}$ or $8 \mathrm{~F}$ catheter sheath. Systemic heparinization was used. According to the position of aneurysm, a $6 \mathrm{~F}$ or $8 \mathrm{~F}$ introducer was connected to a Y-valve together with one or two double-head Y-valves, in the petrous internal carotid artery or vertebral artery at C2 spine level. The best operating angle was selected according to the images reconstructed in a 3D workstation. Under the guidance of a 0.014 micro-guide wire, the two microcatheters were inserted in different tumor locations, heading in different directions. The two microcatheters contain heads in different shapes and angles that vary with irregular aneurysms. The appropriate coils were selected to embolize the aneurysm according to its size and shape. If imaging showed a wide-necked aneurysm that required stent-assisted angioplasty, an $8 \mathrm{~F}$ introducer was 
$\mathbf{A}$

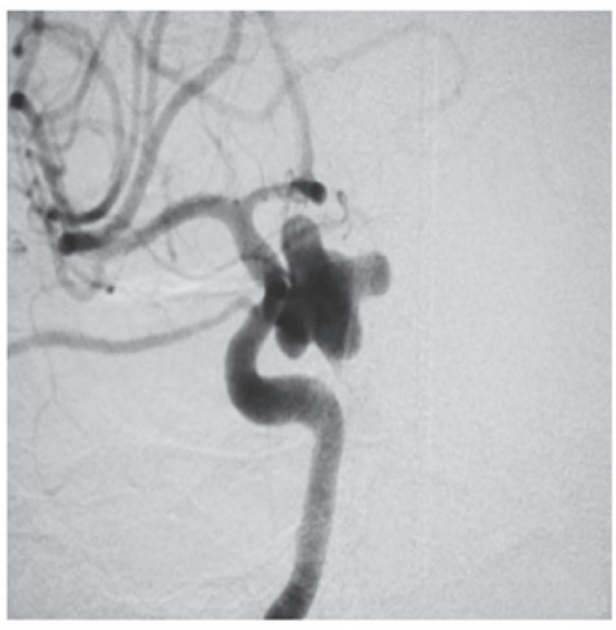

C

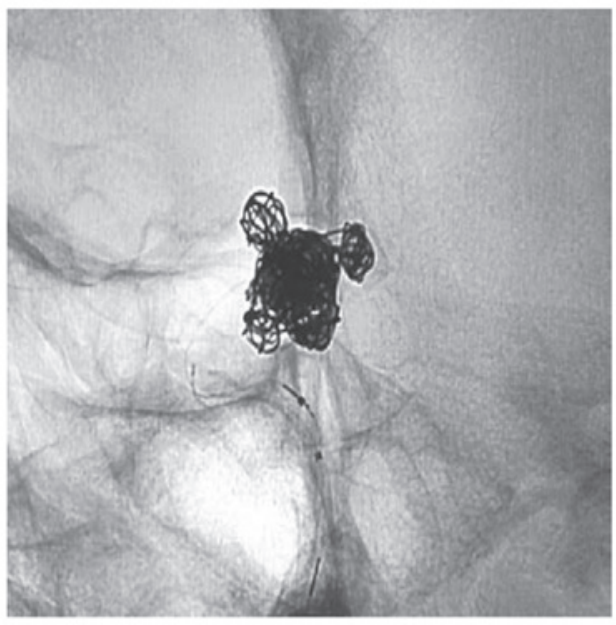

B

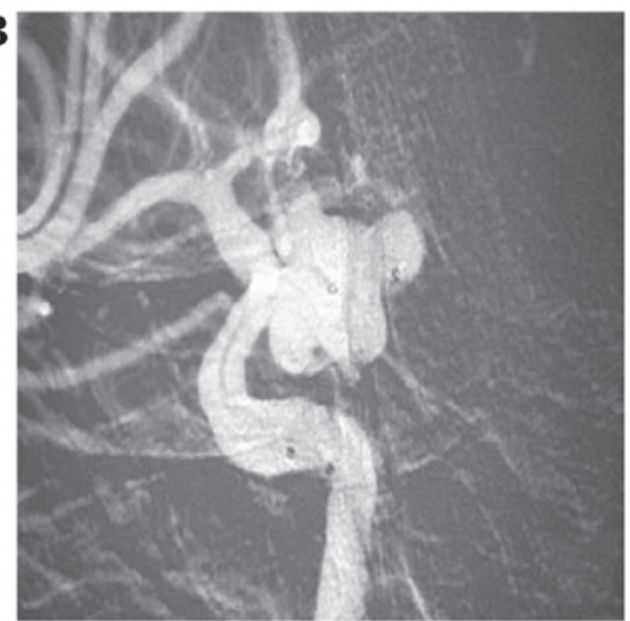

D

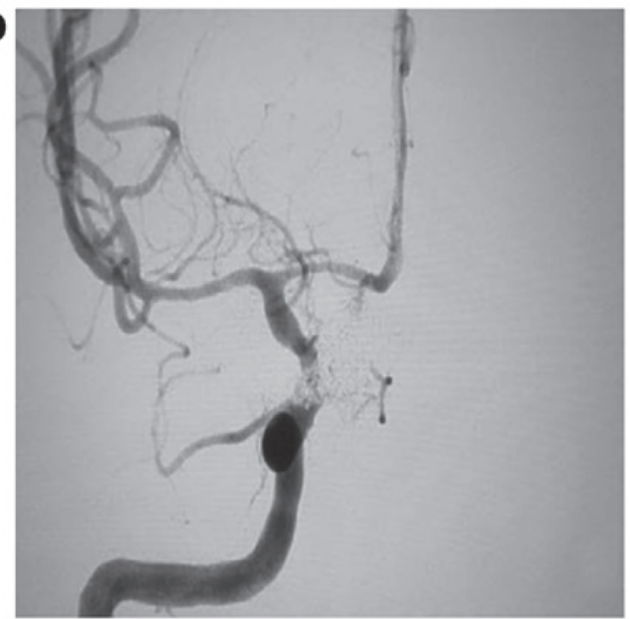

Figure 1. Embolization of the right posterior communicating irregular aneurysm using double microcatheter technique. (A) DSA shows the right posterior communicating cauliflower-like irregular aneurysm, (B) insertion of the two microcatheters into the tumor of the aneurysm in different directions at appropriate surgical angles, (C) embolization with coils of appropriate size alternatively through the tips of the two microcatheters until embolism was satisfactory, and (D) postoperative angiography showing compact embolism of the aneurysm and patency of the parent artery. DSA, digital subtraction angiography.

selected and three microcatheters were used simultaneously. The first coil was woven into a basket based on preset microcatheter varying with the size and shape of the aneurysm. The second coil required the double microcatheter technique for alternate embolization based on the coil shape inside the aneurysm, until it was compactly embolized (Figs. 1 and 2).

Evaluation parameters. Concerning fate of embolization, a failure to detect the tumor and aneurysmal neck completely indicated totally compact embolism. Detection of aneurysmal neck but not tumor indicates near-total embolism while detection of the tumor and aneurysmal neck suggested adequate embolism. At the time of hospital discharge, the modified Rankin Scale score was used to evaluate the prognosis.

\section{Results}

The 37 cases were successfully treated of complicated irregular aneurysm. Embolization was achieved via double microcatheter or stent-assisted double microcatheter techniques. Immediately after the procedure, 35 of the 37 aneurysms were compactly embolized as seen on the arteriogram and two cases were almost completely embolized. After the procedure, the patient did not present any neurological deficits and the general clinical status was good. Clinical evaluation using the Modified Glasgow Outcome Scale revealed good prognosis and all patients recovered without any complication or death. After six months, DSA follow-up showed no recurrence but patent vascular branches in 24 cases.

\section{Discussion}

Advances in interventional cardiology and material science led to intravascular therapy as the primary option for treatment of intracranial aneurysms (3). However, for irregular-shaped and complicated intracranial aneurysms, especially lobulated or angulated aneurysms, residual cavities are often found during embolization (4). Despite intervention with double microcatheter for irregular and complicated aneurysms, few published studies are available. Initially, the double microcatheter technique was used to treat wide-necked complicated aneurysms. In 1998, Baxter et al first reported the use of double microcatheter to embolize intracranial wide-necked aneurysms with satisfactory results (5). Over the years, new interventional techniques have been developed, including various intracranial stents. Currently, fewer cases of wide-necked intracerebral aneurysm are treated using double microcatheters. Since 2013, we used the technique to 

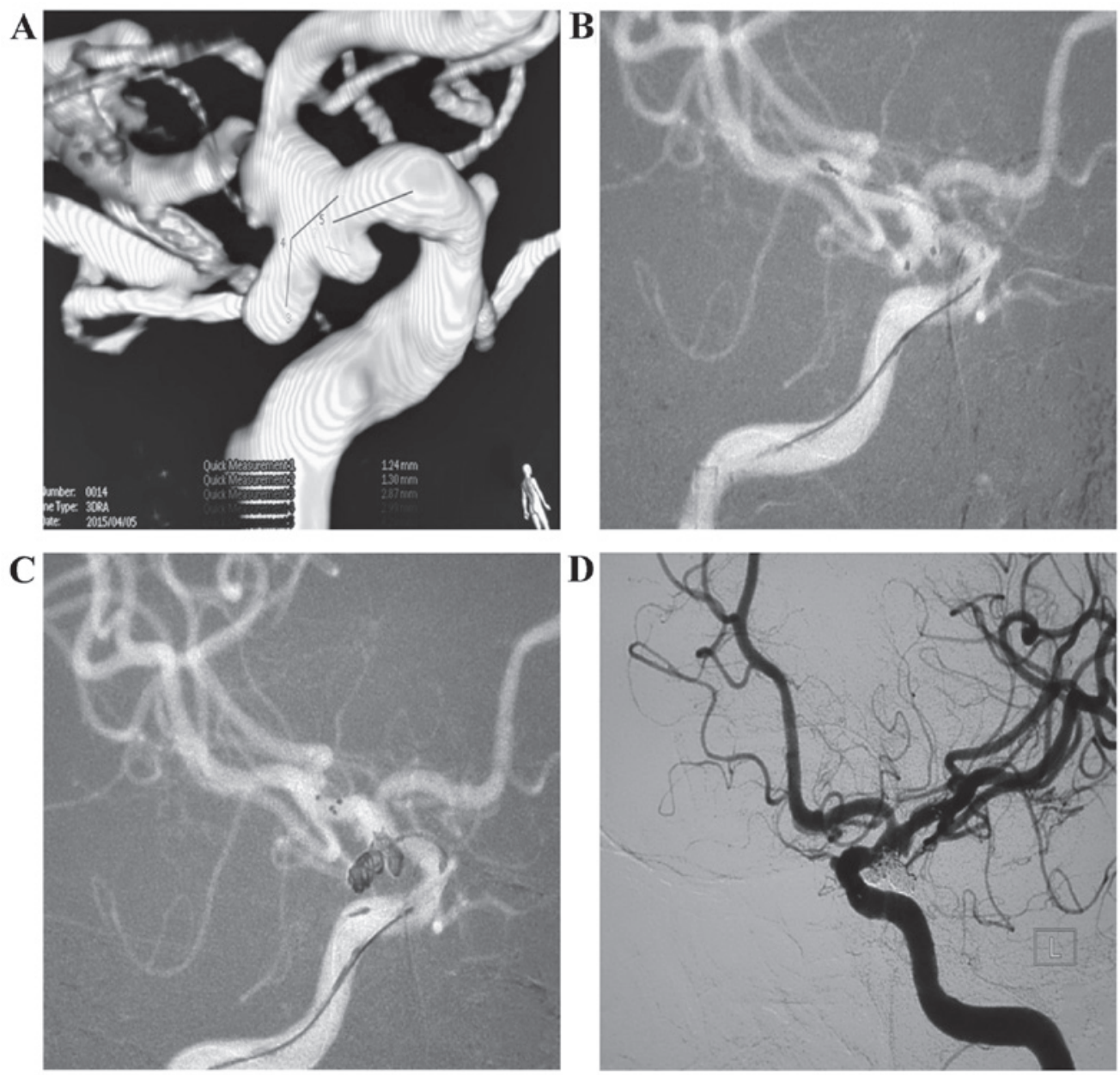

Figure 2. Embolization of the left posterior communicating irregular aneurysm via stenting combined with double microcatheter technique. (A) DSA showing right posterior communicating lobular-shaped irregular aneurysm, (B) implantation of the two microcatheters into the aneurysm in different directions at appropriate surgical angles and insertion of a stent microcatheter into the parent artery, (C) embolization with coils of appropriate size alternatively through the distal tips of the two microcatheters until embolism was satisfactory, and (D) postoperative angiography showing compact embolism of the aneurysm and patency of the parent artery. DSA, digital subtraction angiography.

embolize irregular-shaped aneurysms. During the procedure, the distal tips of the microcatheter are cast into different shapes according to the shape of the aneurysm, and the tips of the two microcatheters are implanted in different positions inside the tumor cavities. Embolization is then carried out using the two microcatheters simultaneously or alternatively, resulting in embolism of intracerebral irregular aneurysms. In case of wide-necked irregular aneurysm, a femoral artery puncture using an $8 \mathrm{~F}$ sheath and $8 \mathrm{~F}$ introducer is feasible along with stenting, concurrently. Our group has successfully performed this technique using double microcatheter or stent-assisted double microcatheter in 37 cases of embolization involving complicated irregular aneurysms. Immediately after the procedure, 35 cases of aneurysm were compactly embolized on the arteriogram and two cases were almost completely embolized. Evaluation using the Modified Glasgow Outcome Scale revealed good prognosis without any complication or death. Follow-up after six months with DSA revealed no recurrence but vascular branches in 24 cases.

In conclusion, the use of double microcatheters has the following advantages (6-11): i) A single 6 F-8 F introducer can be used to control two or three microcatheters simultaneously, which is different from controlling a single microcatheter with difficulty; ii) it obviates the need for reshaping the microcatheter during late-stage embolization of aneurysms due to changes in microcatheter position and the need for securing the position even when the microcatheter is reshaped; iii) stent release at the late stage of the procedure increases the difficulty to reach the ideal position of microcatheter; iv) the rate of compact embolism of aneurysm is greatly increased by the simultaneous resting of the two microcatheters in different positions inside the tumor cavity; and v) by avoiding late-stage reshaping of microcatheter and adjustment of positions, the duration of operation and procedural complications are dramatically reduced.

\section{Acknowledgements}

This study was funded by the Science and Technology Project of Anhui Province in 2015 (grant no. 1506c085017).

\section{References}

1. Lee JY, Seo JH, Cho YD, Kang HS and Han MH: Endovascular treatment of wide-neck intracranial aneurysms using a microcatheter protective technique: results and outcomes in 75 aneurysms.AJNR Am J Neuroradiol 32: 917-922, 2011. 
2. Ding D, Starke RM and Liu KC: Microsurgical strategies following failed endovascular treatment with the pipeline embolization device: case of a giant posterior cerebral artery aneurysm.J Cerebrovasc Endovasc Neurosurg 16: 26-31, 2014.

3. Connolly ES Jr, Rabinstein AA, Carhuapoma JR, Derdeyn CP, Dion J, Higashida RT, Hoh BL, Kirkness CJ, Naidech AM, Ogilvy CS, et al; American Heart Association Stroke Council; Council on Cardiovascular Radiology and Intervention; Council on Cardiovascular Nursing; Council on Cardiovascular Surgery and Anesthesia; Council on Clinical Cardiology: Guidelines for the management of aneurysmal subarachnoid hemorrhage: a guideline for healthcare professionals from the American Heart Association/american Stroke Association. Stroke 43: 1711-1737, 2012.

4. Yu B, Zhang JB, Wang Z, Cai M, Yu XB and Liu YH: Treatment of wide neck aneurysm with coils assisted by hyper compliant balloon. Chinese J Neurol 23: 533-535, 2007 (In Chinese).

5. Baxter BW, Rosso D and Lownie SP: Double microcatheter technique for detachable coil treatment of large, wide-necked intracranial aneurysms. AJNR Am J Neuroradiol 19: 1176-1178, 1998

6. Yu B, Hong Y, Chen LY, Xi Z, Wang MJ, Zheng J, Kan ZY and Liu YH: Treatment of intracranial aneurysms with coils by double micro-catheter technique. Chinese J Neurol 29: 693-69, 2013 (In Chinese).
7. Pu Y, Yang ZX, Yu YT, Shao Y, Miao WF and Yu XR: The application of double microcatheter technique for the treatment of complicated intracranial aneurysms. Chinese J Neurol 31: 31-33, 2015 (In Chinese).

8. Yin L, Huang Y, Wei M, Liang WL, Sun HS and Wang SY: Double microcatheter technique for coiling intracranial aneurysms with unfavorable configurations. Chinese J Contemporary Neurol Neurosurg 13: 216-221, 2013 (In Chinese).

9. Meng L, Zuo LM, Xu SC, Zhao GY and Pang Z: The application of double microcatheter technique for the treatment of ruptured wide neck aneurysm at acute SAH state. Chinese J Critical Care Med 6: 566-568, 2011 (In Chinese).

10. Wen HF, Zhao CX, Li JL, Wang PF and Du JC: Double microcatheter technique for detachable coil treatment of wide-necked and irregular intracranial aneurysms. J Intervent Radiol 21: 890-892, 2012

11. Peng Y, Xuan JG, Yang YL, Wang SN, Xia XW and Chen RH: Application of double microcatheter technique in endovascular $\mathrm{b}$ treatment of intracranial wide-necked aneurysms. Chinese J Cerebrovascular Diseases 7: 371-373, 2009 (In Chinese). 